\title{
Derivation and validation of an exclusive pre-operative risk eval- uation system in HIV-infected patients undergoing surgery
}

\author{
Baochi Liu ${ }^{\mathrm{a}, * \#}$, Tienan Feng ${ }^{\mathrm{b}, *}$, Xiuling Feng ${ }^{\mathrm{c}, *}$, Xiaodong Chen ${ }^{\mathrm{d}}$, Ying Wang ${ }^{\mathrm{d}}$, Yufang Shi $^{\mathrm{d} * \#}$ \\ ${ }^{a}$ Caolang Road No. 2901, Shanghai 201508, China. \\ ${ }^{b}$ Public Health Experimental Center, Shanghai Jiaotong University, Shanghai 200025, China. \\ 'Department of Surgery, Zhengzhou Sixth People Hospital, Zhengzhou450052, China. \\ ${ }^{d}$ Institute of Health Sciences, South Chongqing Road No. 225, Shanghai 200025, China.
}

\section{Abstract}

Background: A broadly applicable scoring tool for risk evaluation in HIV-infected surgical patients is imperative for improving outcomes and patient safety. Because sepsis has been considered as the major cause of mortality for HIV-infected post-surgical patients, the objective of this study was to develop a pre-operative risk stratification model that predicts the incidence of sepsis after surgical operations in HIV-infected patients. Methods: The scoring model was created by using inpatient databases, including 762 HIV-infected patients who underwent various surgical procedures from 2008 to 2014 in the Shanghai Public Health Clinical Center (SPHCC). A risk-point scale was developed with five variables for their predictive value of sepsis incidence using single and multi-factor logistical regressions. This model was validated with a receiver operating characteristic (ROC) curve with a Zhengzhou Sixth People Hospital (ZSPH) dataset (182 HIV-infected cases).

Results: Post-operative sepsis was identified in 256 patients in the SPHCC dataset. The average total scores of the sepsis group and the non-sepsis group were 7.22 and 11.62, respectively. Using the predictive model of multi-factor logistical regression, the sensitivity and specificity were 0.95 and 93.6, respectively. The area under curve (AUC) score was $0.98[0.97,0.99]$. The result was much larger than five single-factor predicting models. In the 30 days after surgery, 15 patients died, and the mortality rate was around 2\% in SPHCC. Postoperative sepsis was identified in 41 patients in the ZSPH dataset, and four sepsis patients died in the 30 days following surgery. The mortality was also around $2 \%$ at ZSPH. The AUC score of the same model was $0.86[0.82$, 0.93].

Conclusion: The risk scoring system with the predictive model has high predictive accuracy. This indicates that it can help surgeons evaluate the incidence and risk of post-surgical sepsis before surgical procedures on HIVinfected patients.

Keywords: Human immunodeficiency virus (HIV); post-operative sepsis; mortality; risk scoring system

\section{INTRODUCTION}

The human immunodeficiency virus type 1 (HIV-1) infection is widely assumed to lead to progressive failure of the immune system (acquired immunodeficiency syndrome, AIDS) and to allow sepsis caused by life-threatening opportunistic infections to occur in patients, especially those

\footnotetext{
*These authors contribute equally to this work.

\#Corresponding authors: Baochi Liu

Fax: +86-21-65981193

Email: liubaochi@shaphc.org

\#Corresponding authors: YufangShi

Mailing address: Institute of Health Sciences, Shanghai Institutes for Biological Sciences, Chinese Academy of Sciences/Shanghai Jiaotong University School of Medicine 225 South Chongqing Road, Shanghai, China 200025

Fax: +86-21-63852655

Email: yufangshi@sibs.ac.cn
}

Received: 20 April 2018 Accepted: 26 June 2018 undergoing surgical operations ${ }^{[1-6]}$. Sepsis is a systemic, deleterious host response to infection leading to severe acute organ dysfunction secondary to a documented or suspected infection. Severe sepsis is a major health care problem affecting millions of people around the world each year, killing one in four (and often more), and increasing in incidence ${ }^{[7-9]}$. HIV-infected patients are related to a higher frequency of severe sepsis and death among patients admitted to the ICU ${ }^{[10]}$. The unacceptably high mortality rates of $30 \%-50 \%$ for severe sepsis continue ${ }^{[9.10]}$ and it can be said that sepsis is a major determinant of outcome in HIV-infected patients ${ }^{[11,12]}$. Therefore, an exclusive and applicable scoring tool for the evaluation of the development of post-operative sepsis is imperative for improving postoperative outcomes in HIV-infected patients.

In this study, we developed a novel pre-operative risk scoring model comprising five variable indicators, including $C D 4+T$ cell count, the incision grade 
used in surgical operations, the surgical grade, the opportunistic infection, and the organ function. To establish the scoring system model, a database of 762 HIV-infected patients in the Shanghai Public Health Clinical Center (SPHCC) was employed. These patients underwent various surgical procedures in the past six years (from 2008 to 2014) for reasons of infection (43.09\%), dysfunction (27.77\%), tumor (21.67\%), and trauma (7.47\%). In the SPHCC dataset, 256 patients had post-operative sepsis. The average total scores of the sepsis group and the non-sepsis group were 7.22 and 11.62, respectively. Using the predicting model of multi-factor logistical regression, the sensitivity and specificity were 0.95 and 93.6. The area under curve (AUC) score was 0.98[0.97, 0.99]. The result, which was much larger than five single-factor predicting models, was validated with a receiver operating characteristic (ROC) curve using a Zhengzhou Sixth People Hospital (ZSPH) dataset of 182 HIVinfected cases with similar reasons for surgery. Postoperative sepsis was identified in 41 patients in the ZSPH dataset. The AUC score of the same model was $0.86[0.82,0.93]$, which indicates that this surgical risk scoring model is effective.

Based on the evaluation indicators, three schemes were proposed and then compared according to the results of calculations. This process helped us confirm the best one with sufficient evidence.

\section{METHODS}

\section{Study Design}

This study was conducted at the SPHCC special center for the treatment of major communicable diseases in Shanghai, China. Clinical documents were retrieved for the date range of January 2010 to December 2014 of 762 patients with a diagnosis of HIV infection and having various surgical procedures to build the predictive model. The clinical documents of 182 patients with the same condition and circumstances from January 2013 to December 2014 at Zhengzhou
Table 1. Basis of risk evaluation

\begin{tabular}{|c|c|c|c|c|}
\hline & 1 & 2 & 3 & 4 \\
\hline CD4 (cells/ $\mu \mathrm{l}$ ) & $>350$ & $(350,200]^{1}$ & $(200,50]$ & $<50$ \\
\hline Incision & Clean & $\begin{array}{l}\text { (Clean, } \\
\text { Pollution) }\end{array}$ & Pollution & Infection \\
\hline Surgical grade & I & II & III & IV \\
\hline $\begin{array}{l}\text { Opportunistic } \\
\text { infection }\end{array}$ & None & Experienced & Mild & Serious \\
\hline Organ function & Normal & Abnormal & Mild & Serious \\
\hline
\end{tabular}

Sixth People Hospital (HSPH) were collected to evaluate the model. All patients were required to undergo routine laboratory examinations before surgery. The 2001 SCCM/ESICM/ACCP/ATS/SIS international sepsis definition was used as a reference ${ }^{[13]}$. In the SPHCC dataset, the ratio of females to males was around 1:7.4. The age range was from 10 to 74 , and the median was 47.2. In the dataset of ZSPH, the ratio of females to males was 1:3, and the age range was 6 to 81 , with the median being 45 . The risk scoring system graded levels of CD4+ T cell counts, incision, surgical grade, opportunistic infection, and organ function into numerical scales of 1-4. The basis of the grading system is listed in Table 1.

\section{Statistical Analysis}

All data were analyzed using SPSS 17.0 (SPSS, Chicago, IL, USA). Descriptive statistics were represented as mean \pm standard deviation when the datasets were of normal distribution. Otherwise, the descriptions were represented as median [95\% CI low, 95\% CI up]. Demographic and available laboratory data were compared in a single-factor analysis according to the sepsis status. Categorical data were compared using the Chi-square test or the Fisher's test. Measurement data were compared with an independent t-test sample, ANOVA, or the rank-sum test when the datasets did not comply with the normal distribution. The binary logistic regression was used to construct the predicting model. In this regression, the positive condition (sepsis status) was marked with 1 , and the negative was marked with 0 . The logistic regression was set as a predictive model whose performance was

Table 2. Comparison between non-sepsis and sepsis groups

\begin{tabular}{|c|c|c|c|c|c|c|}
\hline & non-sepsis & & sepsis & & & \\
\hline Indicators & Description & Mean Rank & Description & Mean Rank & $\mathrm{Z}$ value & P value \\
\hline CD4 Level & $1.81[3.11,0.50]$ & 322.73 & $2.64[4.06,1.23]$ & 501.10 & 11.05 & $<.001$ \\
\hline Incision & $1.69[2.53,0.86]$ & 328.95 & $2.27[3.38,1.17]$ & 488.76 & 10.62 & $<.001$ \\
\hline Surgical grade & $1.51[2.58,0.45]$ & 328.34 & $2.16[3.39,0.93]$ & 489.98 & 10.38 & $<.001$ \\
\hline Opportunistic infection & $1.15[1.71,0.60]$ & 269.39 & $2.52[3.46,1.57]$ & 605.03 & 22.41 & $<.001$ \\
\hline Organ function & $1.06[1.45,0.67]$ & 274.91 & $2.04[2.80,1.27]$ & 592.18 & 22.70 & $<.001$ \\
\hline Sum & $7.22[9.31,5.13]$ & 306.71 & $11.62[14.10,9.15]$ & 646.01 & 19.25 & $<.001$ \\
\hline
\end{tabular}


Table 3. Results of single-factor logistic regression

\begin{tabular}{llll}
\hline Indicators & sensitivity & specificity & accuracy \\
\hline CD4(cells/ $\mu \mathrm{l})$ & 78.1 & 60.2 & 72.1 \\
Incision & 95.3 & 35.9 & 75.4 \\
Surgical grade & 87.8 & 37.9 & 71.1 \\
Opportunistic infection & 85.0 & 95.3 & 88.5 \\
Organ function & 94.1 & 88.7 & 92.3 \\
Sum & 95.1 & 89.8 & 93.3 \\
\hline
\end{tabular}

evaluated by a ROC curve. Statistical significance was set at $\mathrm{p}<0.05$.

\section{Ethical Review and Approval}

The community surveys were conducted and approved by the ethical committees of Shanghai Public Health Clinical Center Affiliated to Fudan University. All received records were anonymized and could not be linked to individual patients. The methods that were carried out in this study were in accordance with the approved guidelines.

\section{RESUITS}

The incidence of post-operation sepsis in the SPHCC dataset from 2010 to 2014 was $34 \%$. In the 30 days

Table 4. Results of multi-grade factors logistic regression

\begin{tabular}{|c|c|c|c|c|}
\hline Indicator & Grade & $\log (O R)$ & S.E. & Pvalue \\
\hline \multirow[t]{4}{*}{$\mathrm{CD} 4+\mathrm{T}$ cell counts } & 1 & 1.00 & 1.00 & 0.00 \\
\hline & 2 & 0.88 & 0.46 & 0.05 \\
\hline & 3 & 1.77 & 0.47 & 0.00 \\
\hline & 4 & 1.60 & 0.70 & 0.02 \\
\hline \multirow[t]{4}{*}{ Incision } & 1 & & & 0.00 \\
\hline & 2 & 1.46 & 0.47 & 0.00 \\
\hline & 3 & 3.81 & 0.63 & 0.00 \\
\hline & 4 & 22.61 & 0.98 & 0.00 \\
\hline \multirow[t]{4}{*}{ Surgical grade } & 1 & 1.00 & 1.00 & 0.00 \\
\hline & 2 & 1.27 & 0.43 & 0.00 \\
\hline & 3 & 1.63 & 0.48 & 0.00 \\
\hline & 4 & 3.62 & 1.92 & 0.06 \\
\hline \multirow[t]{4}{*}{ Opportunistic infection } & 1 & 1.00 & 1.00 & 0.00 \\
\hline & 2 & 2.17 & 0.44 & 0.00 \\
\hline & 3 & 4.92 & 0.87 & 0.00 \\
\hline & 4 & 21.24 & 2.31 & 0.00 \\
\hline \multirow[t]{5}{*}{ Organ function } & 1 & 1.00 & 1.00 & 0.00 \\
\hline & 2 & 2.14 & 0.42 & 0.00 \\
\hline & 3 & 3.73 & 1.25 & 0.00 \\
\hline & 4 & & & \\
\hline & Constant & -6.72 & 0.69 & 0.00 \\
\hline
\end{tabular}

after surgery, 15 sepsis patients died, making the mortality around $2 \%$. The evaluation indicators of the two groups are listed in Table 2. Using the rank-sum test, the indicators of the two groups were significantly different. From the results of the $\mathrm{Z}$ value, in addition to the sum of the five indicators, the differences of opportunistic infection and organ function were greater than other indicators.

Next, we used single-factor logistic regression to construct the risk prediction system. Among the six models, the sum model had the best performance.

The results in Table 4 were calculated using multifactor logistic regression. The indicators were categorical. The first grade of each indicator was set as the reference. Because there were no patients with a serious organ failure condition, we had no data of grade 4 for organ failure. Except for the levels of CD4+ $\mathrm{T}$ cell count, the odds ratio (OR) of other grades to the first grade were generally elevated along with an increase of scoring. When the levels of incision and opportunistic infection were at grade 4 , the OR of sepsis incidence was very high. In our institution, all patients with grade 4 incision or grade 4 opportunistic infection developed sepsis. When the level of CD4+ $T$ cell count reached grade 4 , the incidence of postoperative sepsis was $70.15 \%$. The incidence of surgical grade 4 was $85.71 \%$. Patients whose organ function was scored at grade 4 were not allowed to have surgery. When organ function was scored at grade 3 , the incidence was $92 \%$. The performance of the predictive model using multi-factor logistic regression is described in Figure 1. Except for the specificity, it performed better than other models and had greater sensitivity, specificity, and accuracy. Therefore, this model may help offer valuable information to evaluate the risk of post-surgical sepsis among HIV-infected patients before surgery. According to Figure 1, the model can be used to work out the cutoff value (CV), $\mathrm{CV}=\mathrm{Z} /(1+\mathrm{Z})$, where $\mathrm{Z}=0.68 * \mathrm{CD} 4+1.89^{*}$ Incision $+0.86 *$ Surgical_grade $+2.36^{*}$ Opportunistic_infection $+2.06^{*}$ Organ_function-14.64. When $\mathrm{CV}<0.4$, the probability of developing post-operative sepsis was very low. When $\mathrm{CV}$ was in the interval between 0.4 and 0.6 , the probability of developing post-operative sepsis was uncertain. With $\mathrm{CV}>0.6$, the risk of developing post-operative sepsis was very high. During the perioperative period, medical staff needs to pay more attention to reducing the grade. For example, ART could be employed to increase CD4+ T cell counts, prevent opportunistic infections, and also decrease the operation grade.

The model was validated by the dataset from ZSPH, and the results are depicted in Figure 2. Compared to the training dataset, values for sensitivity, specificity, 


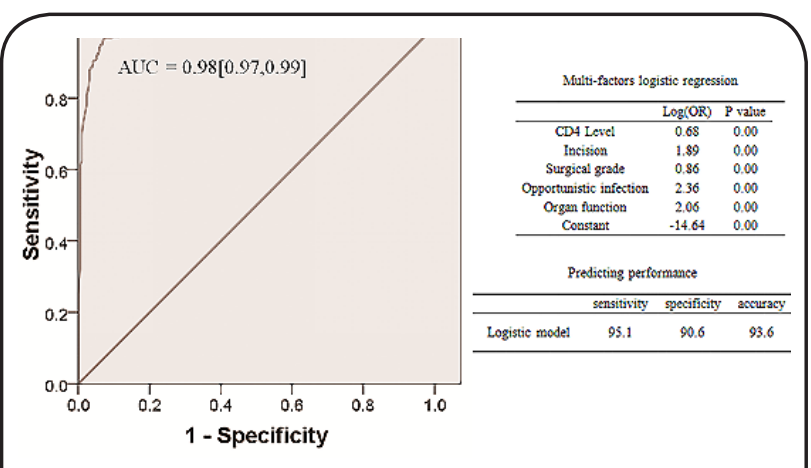

Figure 1. Performance of the multi-factors Predicting model using training data set (SPHCC).

and accuracy were lower, but the performance was still significant. The positive results given by the model indicate that the possibility of developing post-operative sepsis was at least $81 \%$. In addition, the negative results predicted that the possibility of developing post-operative sepsis was not higher than $22 \%$.

\section{DISCUSSION}

There are much more life-threatening issues for HIV-infected patients than for ordinary patients in the recovery period after surgery ${ }^{[14]}$. Based on our long-time clinical experience, we employed CD4+ $\mathrm{T}$ cell counts ${ }^{[15,16]}$, the level of incision, surgical grade, opportunistic infection, and organ function as indicators of post-operative sepsis and graded them into 4 levels.

When CD4+ T cell counts were above 350 cells $/ \mu$, the patient's immune system could still execute its normal function, so we gave this condition a score of 1 under CD4. As the population of CD4+ T cells descended, the immune system became vulnerable. We set the split points at 350,200, and 50 and gave corresponding scores from 2 to 4 , respectively. The incision was also classified into a system of four scores according to its seriousness. A clear incision was given 1 point. If the incision had clear contamination, it was given 2 points. With contamination and infection, 3 and 4 points were given, respectively. The score of surgical grade was calculated according to complications of the surgery. From simple to complex circumstances, it was given from scores of 1 to 4 , accordingly. Opportunistic infection was classified with a score of 4 . With regard to its seriousness, opportunistic infections included no possibility of infections, unobserved historical infections, infections with low probabilities, and obvious signs of infection before surgery, corresponding to 1 to 4 points. The score of organ function was calculated from the comprehensive preoperative examination, including heart, lungs, liver, kidneys, etc., with statuses of normal, abnormality without symptoms, abnormality with slight symptoms, and obvious symptoms of abnormality before surgery, corresponding to scores of 1 to 4 . The surgical risks could be fully evaluated by this scoring system, which had considered comprehensive factors in all parts.

The indicators had different weights in this system. The weight of $\mathrm{CD} 4+\mathrm{T}$ cell count and surgical grade were the lowest, meaning that the link between postoperative sepsis and CD4+ T cell counts was weak, and the link with the surgical grade was also weak, which corresponds with results in other studies ${ }^{[17]}$. The incision was a more important factor needing to be considered before surgery. Among these indicators, the grades of opportunistic infection and organ function were more critical. Small variations of scores from these two indicators could make a great impact on the total surgical risk.

It was reported that the infection and complication rate of HIV-infected patients after surgery was about $55 \%$, and approximately $30 \%$ of those patients died following surgery ${ }^{[18]}$. In our institution, the sepsis incidence was about $40 \%$, and the mortality was less than $2 \%$. Among our patients, the average sum of five indicator scores in non-sepsis and sepsis groups were 7.22 and 11.65 , respectively. When the cutoff value was set at 9.5 , the sensitivity and the specificity were 0.91 and 0.95 , approaching the performance of the logistical predictive model. Therefore, this method can be used as a reference for other groups who intended to perform similar treatments.

In our institution, 15 patients, most of whom had highly complicated surgeries, died from sepsis within 30 days of surgery.

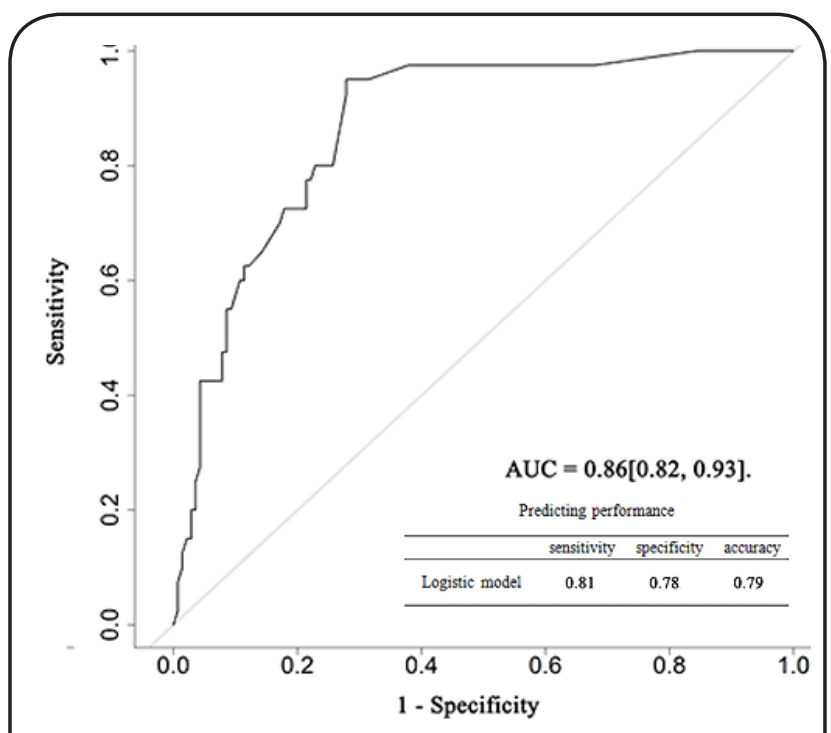

Figure 2. Performance of the Predicting model using the test dataset. 
Because of their particular requirements, HIV-infected patients should be treated in special hospitals. In China, nearly all HIV-infected patients are treated and have surgeries performed in specialized hospitals for epidemic disease. However, there are not enough specialty surgeons to carry out all types of operations. In Europe, Africa, and America, the majority of HIVinfected patients have surgeries performed in general hospitals. Though there are comprehensive disciplines, medical personnel in general hospitals may have insufficient experience in the treatment of HIV-infected patients so that the patients may experience many post-operative complications. Therefore, it is essential to build a platform to resolve this problem. Based on the situation of our institution, patients with CD4+ T cell counts above 350 cells/ $\mu$ l can be treated in general hospitals because they have little difference from normal patients in terms of sepsis incidence. When the CD4+ T cell count is below 200 cells/ $\mu \mathrm{l}$, it is suggested that the patients be treated in specialized hospitals. These patients were prone to opportunistic infections and had higher chances of developing sepsis. During the perioperative period, antiviral, anti-tuberculosis, antifungal, and other similar treatments are essential, and these treatments require the involvement of experienced clinicians. When CD $4+\mathrm{T}$ cell count is between 200 cells $/ \mu$ l and 350 cells $/ \mu$ l, the patient should be treated with antiviral treatments. After the virus load decreases significantly, the patient could undergo surgery in either general or specialized hospitals.

Surgery for HIV-infected patients requires not only the routine preoperative preparation and postoperative treatments but also the prevention of infectious diseases and treatments with antiviral, antituberculosis, and antifungal treatments ${ }^{[19,20]}$. Currently, AIDS has become a chronic infectious disease that can be managed ${ }^{[21,22]}$. Though there are no drugs or preventive vaccines for HIV, AIDS can be dealt with in cases where the person is exposed to HIV accidentally by using emergency treatments such as anti-retroviral preventive treatment ${ }^{[23]}$. Therefore, with essential prevention ${ }^{[20,24,25]}$ it is not necessary to show excessive fear of HIV. When medical staff, especially which are in the emergency room of general hospitals, meet the HIV-infected patient with a low CD4+ T cell count, they can refer to the infectious clinician for consultation, which can help the patient to recover during the perioperative period.

So far, there is not enough research on positive outcomes of post-surgical sepsis in immune dysfunction patients. A risk scoring system for evaluating the conditions for positive surgical sepsis outcomes has not only scientific value but also extensive and profound social significance. The model proposed in this study would serve as a reference with great accuracy. Future studies could include determination of more indicators that can be added to achieve even better accuracy.

\section{ACKNOWLEDGEMENTS}

This work was supported by the National "Eleventh Five" Infectious Disease Special Foundation (2008ZX10001-008).

\section{CONFLICT OF INTEREST}

Conflicts that the editors consider relevant to the content of the manuscript have been disclosed.

\section{REFERENCES}

1. Semprini, A. E., Castagna, C., Ravizza, M., Fiore, S., Savasi, V., Muggiasca, M. L., Grossi, E., Guerra, B., Tibaldi, C., and Scaravelli, G. (1995) The incidence of complications after caesarean section in 156 HIV-positive women. Aids 9, 913-918

2. Liu, B.-C., Zhang, L., Su, J.-S., Tsun, A., and Li, B. (2014) Treatment of postoperative infectious complications in patients with human immunodeficiency virus infection. World journal of emergency medicine 5, 103

3. Donzé, J. D., Ridker, P. M., Finlayson, S. R., and Bates, D. W. (2014) Impact of sepsis on risk of postoperative arterial and venous thromboses: large prospective cohort study. BMJ 349, g5334

4. Lafaurie, M., Dolivo, M., Porcher, R., Rudant, J., Madelaine, I., and Molina, J.-M. (2005) Treatment of facial lipoatrophy with intradermal injections of polylactic acid in HIV-infected patients. JAIDS Journal of Acquired Immune Deficiency Syndromes 38, 393-398

5. Weiss, E. G., and Wexner, S. D. (1995) Surgery for anal lesions in HIV-infected patients. Annals of medicine 27, 467-475

6. Stock, P. G., Barin, B., Murphy, B., Hanto, D., Diego, J. M., Light, J., Davis, C., Blumberg, E., Simon, D., and Subramanian, A. (2010) Outcomes of kidney transplantation in HIV-infected recipients. New England Journal of Medicine 363, 2004-2014

7. Dellinger, R. P., Levy, M. M., Rhodes, A., Annane, D., Gerlach, H., Opal, S. M., Sevransky, J. E., Sprung, C. L., Douglas, I. S., and Jaeschke, R. (2013) Surviving Sepsis Campaign: international guidelines for management of severe sepsis and septic shock, 2012. Intensive care medicine 39, 165-228

8. Dombrovskiy, V. Y., Martin, A. A., Sunderram, J., and Paz, H. L. (2007) Rapid increase in hospitalization and mortality rates for severe sepsis in the United States: A trend analysis from 1993 to 2003*. Critical care medicine 35, 1244-1250

9. Angus, D. C., Linde-Zwirble, W. T., Lidicker, J., 
Clermont, G., Carcillo, J., and Pinsky, M. R. (2001) Epidemiology of severe sepsis in the United States: analysis of incidence, outcome, and associated costs of care. Critical care medicine 29, 1303-1310

10. Medrano, J., Álvaro-Meca, A., Boyer, A., JiménezSousa, M. A., and Resino, S. (2014) Mortality of patients infected with HIV in the intensive care unit (2005 through 2010): significant role of chronic hepatitis $\mathrm{C}$ and severe sepsis. Critical Care 18, 475

11. Japiassú, A. M., Amâncio, R. T., Mesquita, E. C., Medeiros, D. M., Bernal, H. B., Nunes, E. P., Luz, P. M., Grinsztejn, B., and Bozza, F. A. (2010) Sepsis is a major determinant of outcome in critically ill HIV/ AIDS patients. Crit Care 14, R152

12. Jacob, S. T., Moore, C. C., Banura, P., Pinkerton, R., Meya, D., Opendi, P., Reynolds, S. J., KenyaMugisha, N., Mayanja-Kizza, H., and Scheld, W. M. (2009) Severe sepsis in two Ugandan hospitals: a prospective observational study of management and outcomes in a predominantly HIV-1 infected population. PLoS One 4, e7782

13. Levy, M. M., Fink, M. P., Marshall, J. C., Abraham, E., Angus, D., Cook, D., Cohen, J., Opal, S. M., Vincent, J.L., and Ramsay, G. (2003) 2001 sccm/esicm/accp/ ats/sis international sepsis definitions conference. Intensive care medicine 29, 530-538

14. Su, J., Tsun, A., Zhang, L., Xia, X., Li, B., Guo, R., and Liu, B. (2013) Preoperative risk factors influencing the incidence of postoperative sepsis in human immunodeficiency virus-infected patients: a retrospective cohort study. World journal of surgery 37, 774-779

15. Deneve, J. L., Shantha, J. G., Page, A. J., Wyrzykowski, A. D., Rozycki, G. S., and Feliciano, D. V. (2010) CD4 count is predictive of outcome in HIV-positive patients undergoing abdominal operations. The American Journal of Surgery 200, 694-700

16. King Jr, J. T., Perkal, M. F., Rosenthal, R. A., Gordon, A. J., Crystal, S., Rodriguez-Barradas, M. C., Butt, A. A., Gibert, C. L., Rimland, D., and Simberkoff, M. S. (2015) Thirty-Day Postoperative Mortality Among Individuals With HIV Infection Receiving Antiretroviral Therapy and Procedure-Matched,
Uninfected Comparators. JAMA surgery

17. Čačala, S., Mafana, E., Thomson, S., and Smith, A. (2006) Prevalence of HIV status and CD4 counts in a surgical cohort: their relationship to clinical outcome. Annals of the Royal College of Surgeons of England 88, 46

18. Kedir, M. (2008) Seroprevalence, pattern and outcome of HIV/AIDS among surgical patients at Gondar University Hospital. Ethiopian medical journal 46, 15-18

19. Liu, B., Zhang, L., Guo, R., Su, J., Li, L., and Si, Y. (2012) Anti-infective treatment in HIV-infected patients during perioperative period. AIDS Res Ther 9, 36

20. Zhang, L., Liu, B.-C., Zhang, X.-Y., Li, L., Xia, X.-J., and Guo, R.-Z. (2012) Prevention and treatment of surgical site infection in HIV-infected patients. BMC infectious diseases 12, 115

21. Chirch, L. M., Hasham, M., and Kuchel, G. A. (2014) HIV and aging: a clinical journey from Koch's postulate to the chronic disease model and the contribution of geriatric syndromes. Current Opinion in HIV and AIDS 9, 405-411

22. Baker, D., Pell, C., and Donovan, B. (2014) HIV as a chronic disease: optimising outcomes. Medicine Today 15, 16-26

23. Cohen, M. S., Chen, Y. Q., McCauley, M., Gamble, T., Hosseinipour, M. C., Kumarasamy, N., Hakim, J. G., Kumwenda, J., Grinsztejn, B., and Pilotto, J. H. (2011) Prevention of HIV-1 infection with early antiretroviral therapy. New England journal of medicine 365, 493-505

24. Islam, J., Clarke, D. L., and Thomson, S. R. (2014) Lessons from emergency laparotomy for abdominal tuberculosis in the HIV/AIDS era. South African Journal of Surgery 52, 10-12

25. Leow, J. J., Groen, R. S., Bae, J. Y., Adisa, C. A., Kingham, T. P., and Kushner, A. L. (2012) Scarcity of healthcare worker protection in eight low-and middle-income countries: surgery and the risk of HIV and other bloodborne pathogens. Tropical Medicine \& International Health 17, 397-401 